PROCEEDINGS OF THE

AMERICAN MATHEMATICAL SOCIETY

Volume 132, Number 4, Pages 1177-1180

S 0002-9939(03)07223-X

Article electronically published on August 28, 2003

\title{
MINIMAL VECTORS IN ARBITRARY BANACH SPACES
}

\author{
VLADIMIR G. TROITSKY
}

(Communicated by N. Tomczak-Jaegermann)

\begin{abstract}
We extend the method of minimal vectors to arbitrary Banach spaces. It is proved, by a variant of the method, that certain quasinilpotent operators on arbitrary Banach spaces have hyperinvariant subspaces.
\end{abstract}

The method of minimal vectors was introduced by Ansari and Enflo in AE98 in order to prove the existence of invariant subspaces for certain classes of operators on a Hilbert space. Pearcy used it in $[\mathrm{P}]$ to prove a version of Lomonosov's theorem. Androulakis in [A] adapted the technique to super-reflexive Banach spaces. In [CPS] the method was independently generalized to reflexive Banach spaces. There has been hope that this technique could provide a positive solution to the invariant subspace problem for these spaces. In this note we present a version of the method of minimal vectors (based on $[\mathrm{A}]$ ) that works for arbitrary Banach spaces. In particular, it applies in the spaces where there are known examples of operators without invariant subspaces, e.g., E76, E87, R84, [R85. This shows that the method of minimal vectors alone cannot solve the invariant subspace problem for "good" spaces.

Suppose that $X$ is a Banach space. For simplicity, we assume that $X$ is a real Banach space, though the results can be adapted to the complex case in a straightforward manner. In the following, $B\left(x_{0}, \varepsilon\right)$ stands for the closed ball of radius $\varepsilon$ centered at $x_{0}$ while $B^{\circ}\left(x_{0}, \varepsilon\right)$ stands for the open ball, and $S\left(x_{0}, \varepsilon\right)$ stands for the corresponding sphere.

Let $Q$ be a bounded operator on $X$. Since we will be interested in the hyperinvariant subspaces of $Q$, we can assume, without loss of generality, that $Q$ is one-to-one and has dense range, since otherwise $\operatorname{ker} Q$ or $\overline{\operatorname{Range} Q}$ would be hyperinvariant for $Q$. By $\{Q\}^{\prime}$ we denote the commutant of $Q$.

Fix a point $x_{0} \neq 0$ in $X$ and a positive real $\varepsilon<\left\|x_{0}\right\|$. Let $K=Q^{-1} B\left(x_{0}, \varepsilon\right)$. Clearly, $K$ is a convex closed set. Note that $0 \notin K$ and $K \neq \varnothing$ because $Q$ has dense range. Let $d=\inf _{K}\|z\|$. Then $d>0$. It is observed in [AE98], A] that if $X$ is reflexive, then there exists $z \in K$ with $\|z\|=d$. Such a vector is called a minimal vector for $x_{0}, \varepsilon$ and $Q$. Even without the reflexivity condition, however, one can always find $y \in K$ with $\|y\| \leq 2 d$; such a $y$ will be referred to as a 2-minimal vector for $x_{0}, \varepsilon$ and $Q$.

Received by the editors November 27, 2002 and, in revised form, December 22, 2002.

2000 Mathematics Subject Classification. Primary 47A15.

Key words and phrases. Invariant subspace, minimal vector.

(C)2003 American Mathematical Society 
The set $K \cap B(0, d)$ is the set of all minimal vectors; in general, this set may be empty. If $z$ is a minimal vector, since $z \in K=Q^{-1} B\left(x_{0}, \varepsilon\right)$, then $Q z \in B\left(x_{0}, \varepsilon\right)$. Since $z$ is an element of minimal norm in $K$, then, in fact, $Q z \in S\left(x_{0}, \varepsilon\right)$. Since $Q$ is one-to-one, we have

$$
Q B(0, d) \cap B\left(x_{0}, \varepsilon\right)=Q(B(0, d) \cap K) \subseteq S\left(x_{0}, \varepsilon\right) .
$$

It follows that $Q B(0, d)$ and $B^{\circ}\left(x_{0}, \varepsilon\right)$ are two disjoint convex sets. Since one of them has nonempty interior, they can be separated by a continuous linear functional (see, e.g., AB99, Theorem 5.5]). That is, there exists a functional $f$ with $\|f\|=1$ and a positive real $c$ such that $f_{\mid Q B(0, d)} \leq c$ and $f_{\mid B^{\circ}\left(x_{0}, \varepsilon\right)} \geq c$. By continuity, $f_{\mid B\left(x_{0}, \varepsilon\right)} \geq c$. We say that $f$ is a minimal functional for $x_{0}$, $\varepsilon$, and $Q$.

We claim that $f\left(x_{0}\right) \geq \varepsilon$. Indeed, for every $x$ with $\|x\| \leq 1$ we have $x_{0}-\varepsilon x \in$ $B\left(x_{0}, \varepsilon\right)$. It follows that $f\left(x_{0}-\varepsilon x\right) \geq c$, so that $f\left(x_{0}\right) \geq c+\varepsilon f(x)$. Taking sup over all $x$ with $\|x\| \leq 1$ we get $f\left(x_{0}\right) \geq c+\varepsilon\|f\| \geq \varepsilon$.

Observe that the hyperplane $Q^{*} f=c$ separates $K$ and $B(0, d)$. Indeed, if $z \in B(0, d)$, then $\left(Q^{*} f\right)(z)=f(Q z) \leq c$, and if $z \in K$, then $Q z \in B\left(x_{0}, \varepsilon\right)$ so that $\left(Q^{*} f\right)(z)=f(Q z) \geq c$. For every $z$ with $\|z\| \leq 1$ we have $d z \in B(0, d)$, so that $\left(Q^{*} f\right)(d z) \leq c$. It follows that $\left\|Q^{*} f\right\| \leq \frac{c}{d}$. On the other hand, for every $\delta>0$ there exists $z \in K$ with $\|z\| \leq d+\delta$. Then $\left(Q^{*} f\right)(z) \geq c \geq \frac{c}{d+\delta}\|z\|$, whence $\left\|Q^{*} f\right\| \geq \frac{c}{d+\delta}$. It follows that $\left\|Q^{*} f\right\|=\frac{c}{d}$. For every $z \in K$ we have $\left(Q^{*} f\right)(z) \geq c=d\left\|Q^{*} f\right\|$. In particular, if $y$ is a 2 -minimal vector, then

$$
\left(Q^{*} f\right)(y) \geq \frac{1}{2}\left\|Q^{*} f\right\|\|y\| \text {. }
$$

We proceed to the main theorem.

Theorem. Let $Q$ be a quasinilpotent operator on a Banach space $X$, and suppose that there exists a closed ball $B$ such that $0 \notin B$ and for every sequence $\left(x_{n}\right)$ in $B$ there is a subsequence $\left(x_{n_{i}}\right)$ and a sequence $\left(K_{i}\right)$ in $\{Q\}^{\prime}$ such that $\left\|K_{i}\right\| \leq 1$ and $\left(K_{i} x_{n_{i}}\right)$ converges in norm to a nonzero vector. Then $Q$ has a hyperinvariant subspace.

Remark. The hypothesis of the theorem is slightly weaker than the condition $(*)$ in $\left[\mathrm{A}\right.$, where it is required that for every $\varepsilon \in(0,1)$, there exists $x_{0}$ of norm one such that the ball $B\left(x_{0}, \varepsilon\right)$ satisfies the rest of the condition.

Proof. Without loss of generality, $Q$ is one-to-one and has dense range. Let $x_{0} \neq 0$ and $\varepsilon \in\left(0,\left\|x_{0}\right\|\right)$ be such that $B=B\left(x_{0}, \varepsilon\right)$. For every $n \geq 1$ choose a 2 -minimal vector $y_{n}$ and a minimal functional $f_{n}$ for $x_{0}, \varepsilon$, and $Q^{n}$.

Since $Q$ is quasinilpotent, there is a subsequence $\left(y_{n_{i}}\right)$ such that $\frac{\left\|y_{n_{i}-1}\right\|}{\left\|y_{n_{i}}\right\|} \rightarrow 0$. Indeed, otherwise there would exist $\delta>0$ such that $\frac{\left\|y_{n-1}\right\|}{\left\|y_{n}\right\|}>\delta$ for all $n$, so that $\left\|y_{1}\right\| \geq \delta\left\|y_{2}\right\| \geq \cdots \geq \delta^{n}\left\|y_{n+1}\right\|$. Since $Q^{n} y_{n+1} \in Q^{-1} B$, we have

$$
\left\|Q^{n} y_{n+1}\right\| \geq d \geq \frac{\left\|y_{1}\right\|}{2} \geq \frac{\delta^{n}}{2}\left\|y_{n+1}\right\| \text {. }
$$

It follows that $\left\|Q^{n}\right\| \geq \delta^{n} / 2$, which contradicts the quasinilpotence of $Q$.

Since $\left\|f_{n_{i}}\right\|=1$ for all $i$, we can assume (by passing to a further subsequence), that $\left(f_{n_{i}}\right)$ weak*-converges to some $g \in X^{*}$. Since $f_{n}\left(x_{0}\right) \geq \varepsilon$ for all $n$, it follows that $g\left(x_{0}\right) \geq \varepsilon$. In particular, $g \neq 0$.

Consider the sequence $\left(Q^{n_{i}-1} y_{n_{i}-1}\right)_{i=1}^{\infty}$. It is contained in $B$, so that by passing to yet a further subsequence, if necessary, we find a sequence $\left(K_{i}\right)$ in $\{Q\}^{\prime}$ such 
that $\left\|K_{i}\right\| \leq 1$ and $K_{i} Q^{n_{i}-1} y_{n_{i}-1}$ converges in norm to some $w \neq 0$. Put

$$
Y=\{Q\}^{\prime} Q w=\left\{T Q w \mid T \in\{Q\}^{\prime}\right\} .
$$

One can easily verify that $Y$ is a linear subspace of $X$ invariant under $\{Q\}^{\prime}$. Notice that $Y$ is nontrivial because $Q$ is one-to-one and $0 \neq Q w \in Y$. We will show that $Y \subseteq \operatorname{ker} g$, so that $\bar{Y}$ is a proper $Q$-hyperinvariant subspace.

Take $T \in\{Q\}^{\prime}$; we will show that $g(T Q w)=0$. It follows from (1) that $\left(Q^{* n_{i}} f_{n_{i}}\right)\left(y_{n_{i}}\right) \neq 0$ for every $i$, so that $X=\operatorname{span}\left\{y_{n_{i}}\right\} \oplus \operatorname{ker}\left(Q^{* n_{i}} f_{n_{i}}\right)$. Then one can write $T K_{i} y_{n_{i}-1}=\alpha_{i} y_{n_{i}}+r_{i}$, where $\alpha_{i}$ is a scalar and $r_{i} \in \operatorname{ker}\left(Q^{* n_{i}} f_{n_{i}}\right)$. We claim that $\alpha_{i} \rightarrow 0$. Indeed,

$$
\left(Q^{* n_{i}} f_{n_{i}}\right)\left(T K_{i} y_{n_{i}-1}\right)=\alpha_{i}\left(Q^{* n_{i}} f_{n_{i}}\right)\left(y_{n_{i}}\right),
$$

and, combining this with (11), we get

$$
\left|\left(Q^{* n_{i}} f_{n_{i}}\right)\left(T K_{i} y_{n_{i}-1}\right)\right| \geq \frac{\left|\alpha_{i}\right|}{2}\left\|Q^{* n_{i}} f_{n_{i}}\right\|\left\|y_{n_{i}}\right\| .
$$

On the other hand,

$$
\left|\left(Q^{* n_{i}} f_{n_{i}}\right)\left(T K_{i} y_{n_{i}-1}\right)\right| \leq\left\|Q^{* n_{i}} f_{n_{i}}\right\| \cdot\|T\| \cdot\left\|y_{n_{i}-1}\right\| .
$$

It follows from (3) and (4) that

$$
\left|\alpha_{i}\right| \leq 2\|T\| \frac{\left\|y_{n_{i}-1}\right\|}{\left\|y_{n_{i}}\right\|} \rightarrow 0 .
$$

Then (2) yields that

$$
\begin{aligned}
\left|f_{n_{i}}\left(Q^{n_{i}} T K_{i} y_{n_{i}-1}\right)\right|= & \left|\alpha_{i} f_{n_{i}}\left(Q^{n_{i}} y_{n_{i}}\right)\right| \\
& \leq\left|\alpha_{i}\right| \cdot\left\|f_{n_{i}}\right\| \cdot\left\|Q^{n_{i}} y_{n_{i}}\right\| \leq\left|\alpha_{i}\right| \cdot 1 \cdot\left(\left\|x_{0}\right\|+\varepsilon\right) \rightarrow 0,
\end{aligned}
$$

so that $f_{n_{i}}\left(Q^{n_{i}} T K_{i} y_{n_{i}-1}\right) \rightarrow 0$. On the other hand, since $T, K_{i} \in\{Q\}^{\prime}$ we have

$$
Q^{n_{i}} T K_{i} y_{n_{i}-1}=T Q K_{i} Q^{n_{i}-1} y_{n_{i}-1} \rightarrow T Q w
$$

in norm, while $f_{n_{i}} \stackrel{w^{*}}{\longrightarrow} g$, so that $f_{n_{i}}\left(Q^{n_{i}} T K_{i} y_{n_{i}-1}\right) \rightarrow g(T Q w)$. Hence, $g(T Q w)=$ 0 .

Clearly, the argument will work as well for $\lambda$-minimal vectors for any $\lambda>1$.

Suppose that $Q$ is a quasinilpotent operator commuting with a compact operator $K$. Then $Q$ satisfies the hypothesis of the theorem. Indeed, without loss of generality, $\|K\|=1$. Fixing $\varepsilon=\frac{1}{3}$, there exists $x_{0}$ with $\left\|x_{0}\right\|=1$ such that $\left\|K x_{0}\right\| \geq \frac{2}{3}$ and $0 \notin K B\left(x_{0}, \varepsilon\right)$. For every sequence $\left(x_{n}\right)$ in $B\left(x_{0}, \varepsilon\right)$, the sequence $\left(K x_{n}\right)$ has a convergent subsequence $\left(K x_{n_{i}}\right)$. Take $K_{i}=K$ for all $i$; since $0 \notin K B\left(x_{0}, \varepsilon\right)$ we have $\lim _{i} K_{i} x_{n_{i}} \neq 0$. It follows from the theorem that if $Q$ is a quasinilpotent operator on a real or complex Banach space commuting with a nonzero compact operator, then $Q$ has a hyperinvariant subspace. This fact is not new though: for complex Banach spaces it is a special case of the celebrated Lomonosov's theorem [L73], and for real Banach spaces it follows from Theorem 2 of $\mathrm{H} 81$. 


\section{ACKNOWLEDGEMENTS}

Thanks are due to A. Litvak, N. Tomczak-Jaegermann, and R. Vershynin for their interest in the work and helpful suggestions. The author would also like to thank the Department of Mathematics of the University of Alberta for its support and hospitality.

\section{REFERENCES}

[AB99] C. D. Aliprantis and K. C. Border, Infinite-dimensional analysis, a hitchhiker's guide, second edition, Springer-Verlag, Berlin, 1999. MR 2000k:46001

[AE98] S. Ansari and P. Enflo, Extremal vectors and invariant subspaces, Trans. Amer. Math. Soc. 350 (1998), no. 2, 539-558. MR 98d:47019

[A] G. Androulakis, A note on the method of minimal vectors, Trends in Banach spaces and operator theory, Contemporary Mathematics (A. Kaminska, editor), Vol. 321, Amer. Math. Soc., Providence, RI, 2003, pp. 29-36.

[CPS] I. Chalendar, J. R. Partington, and M. Smith, Approximation in reflexive Banach spaces and applications to the invariant subspace problem, Proc. Amer. Math. Soc. (2004), to appear.

[E76] P. Enflo, On the invariant subspace problem in Banach spaces, Séminaire MaureySchwartz (1975-1976) Espaces $L^{p}$, applications radonifiantes et géométrie des espaces de Banach, Exp. Nos. 14-15, Centre Math., École Polytech., Palaiseau, 1976, pp. 1-7. MR 57:13530

[E87] - On the invariant subspace problem for Banach spaces, Acta Math. 158 (1987), no. 3-4, 213-313. MR 88j:47006

[H81] N. D. Hooker, Lomonosov's hyperinvariant subspace theorem for real spaces, Math. Proc. Cambridge Philos. Soc. 89 (1981), no. 1, 129-133. MR 84a:47009

[L73] V. I. Lomonosov, Invariant subspaces of the family of operators that commute with a completely continuous operator, Funkcional. Anal. i Priložen. 7 (1973), no. 3, 55-56. MR 54:8319

[P] C. Pearcy, On technique of Enflo, Proc. Amer. Math. Soc., to appear.

[R84] C. J. Read, A solution to the invariant subspace problem, Bull. London Math. Soc. 16 (1984), no. 4, 337-401. MR 86f:47005

[R85] - A solution to the invariant subspace problem on the space $\ell_{1}$, Bull. London Math. Soc. 17 (1985), no. 4, 305-317. MR 87e:47013

[RR73] H. Radjavi and P. Rosenthal, Invariant subspaces, Ergebnisse der Mathematik und ihrer Grenzgebiete, Band 77, Springer-Verlag, New York, 1973. MR 51:3924

Department of Mathematics, University of Alberta, Edmonton, AB, T6G 2G1 Canada

E-mail address: vtroitsky@math.ualberta.ca 